\title{
Irrational Beliefs and Psychological Distress: A Meta-Analysis
}

\author{
Andreea Vîslă ${ }^{a, b}$ Christoph Flückiger ${ }^{b, c}$ Martin grosse Holtforth ${ }^{b-d}$ \\ Daniel David ${ }^{\mathrm{a}, \mathrm{e}}$ \\ aDepartment of Clinical Psychology and Psychotherapy, Babeş-Bolyai University, Cluj-Napoca, Romania; \\ ${ }^{b}$ Department of Psychology, University of Zurich, Zurich, 'Department of Psychology, University of Bern, and

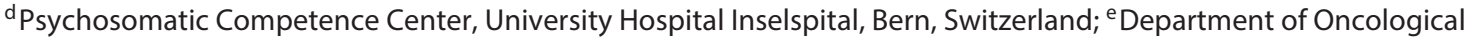 \\ Sciences, Icahn School of Medicine at Mount Sinai, New York, N.Y., USA
}

\section{Key Words}

Irrational beliefs · Meta-analysis $\cdot$ Psychological distress

\begin{abstract}
Background: Since the cognitive revolution of the early 1950s, cognitions have been discussed as central components in the understanding and treatment of mental illnesses. Even though there is an extensive literature on the association between therapy-related cognitions such as irrational beliefs and psychological distress over the past 60 years, there is little meta-analytical knowledge about the nature of this association. Methods: The relationship between irrational beliefs and distress was examined based on a systematic review that included 100 independent samples, gathered in 83 primary studies, using a random-effect model. The overall effects as well as potential moderators were examined: (a) distress measure, (b) irrational belief measure, (c) irrational belief type, (d) method of assessment of distress, (e) nature of irrational beliefs, $(\mathrm{f})$ time lag between irrational beliefs and distress assessment, (g) nature of stressful events, (h) sample characteristics (i.e. age, gender, income, and educational, marital, occupational and clinical status), (i) developer/validator status of the author(s), and (k) publication year and
\end{abstract}

\section{KARGER}

E-Mail karger@karger.com

www.karger.com/pps country. Results: Overall, irrational beliefs were positively associated with various types of distress, such as general distress, anxiety, depression, anger, and guilt (omnibus: $r=$ 0.38). The following variables were significant moderators of the relationship between the intensity of irrational beliefs and the level of distress: irrational belief measure and type, stressful event, age, educational and clinical status, and developer/validator status of the author. Conclusions: Irrational beliefs and distress are moderately connected to each other; this relationship remains significant even after controlling for several potential covariates.

(c) 2015 S. Karger AG, Basel

\section{Introduction}

Given the high prevalence of mental disorders in the population and the considerable economic burden associated, it is imperative to disseminate effective treatments that have enduring effects for patients over time [1]. However, in order to establish and disseminate psychotherapeutic treatments, there is not only a claim to investigate their efficacy/effectiveness, but also a demand for a clear understanding of their underlying theory and psy-

Andreea Vîslă

Department of Clinical Psychology and Psychotherapy, Babeş-Bolyai University Republicii 37

RO-400015 Cluj-Napoca (Romania)

E-Mail andreea.visla@gmail.com 
chological mechanisms [2, 3]. Therefore, investigating how fundamental psychological mechanisms postulated to produce change in these treatments relate to psychological distress becomes a necessary step towards validating these treatments.

Irrational beliefs are lasting constructs used over the past decades and co-developed in the Rational Emotive Behavior Therapy (REBT), an early cognitive-behavioral framework for the treatment of mental disorders [4]. After initially proposing 11 irrational or evaluative belief types [5], subsequent developments in REBT [6] assigned these types of irrational beliefs to four categories: demandingness (i.e. absolutistic/inflexible requirements), awfulizing (or catastrophizing), frustration intolerance (or low frustration tolerance) and global evaluation of one's own person (self-downing), other persons (other-downing) and/or the life situation (lifedowning). In stressful activating situations, irrational beliefs are hypothesized to engender dysfunctional emotions [7]. Another main approach in clinical psychology focuses on the role of automatic thoughts and cognitive schemas (or representations) in generating distress [8]. It has been shown that while representations contribute to emotion, only evaluations (i.e. how relevant the event is for the individual) have a direct impact on emotional response $[9,10]$.

Over the years, many studies investigated REBT and helped to refine the theory and practice of REBT based on a large number of diverse narrative reviews of empirical research $[11,12]$. However, thus far, the quantitative findings associated with these reviews have not been summarized using meta-analytic methods. Thus, the major aim of the present investigation was to examine the quantitative findings regarding the correlational relationship between irrational beliefs and various types of psychological distress using meta-analytic methods. Furthermore, we aimed to investigate the role of the following variables as potential moderators of the relationship between the level of irrational beliefs and the intensity of psychological distress: (a) distress measure; (b) irrational belief measure; (c) irrational belief type; (d) method of assessment of distress (i.e. self-report vs. observer report); (e) nature of irrational beliefs (i.e. general vs. specific, and naturally varying vs. manipulated); (f) time lag between irrational beliefs and distress assessment; (g) nature of stressful events (i.e. present vs. absent, real/naturalistic vs. experimentally induced, personally relevant vs. not personally relevant); (h) sample characteristics (e.g. age, gender, income, and educational, marital, occupational and clinical status); (i) devel-

Irrational Beliefs and Psychological Distress oper/validator status of the author(s), and (k) publication year and country.

We expect distinct patterns of association between irrational beliefs and distress, considering that some distress measures contain mostly emotional items, while others contain a mixture of emotional, behavioral and somatic items [13]. Second, we expect to obtain a significantly stronger irrational belief-distress relationship when irrational beliefs were measured using scales that contain emotional items (e.g. the Irrational Beliefs Test, IBT [14]), as opposed to newer scales containing only cognitive items (e.g. the Survey of Personal Beliefs, SPB [15]; the Attitude and Belief Scale, ABS [16], and the Irrational Belief Scale, IBS [17]). In addition, reliability indices of the different measures might also impact this relationship [18]. Third, we expect that the various irrational belief types (e.g. demandingness and awfulizing) would show significantly different associations with different types of psychological distress (e.g. anxiety and depression [19]). Fourth, we expect that the strength of the irrational belief-distress association will differ depending on the observational perspective (i.e. self-report vs. clinician report [20]). Fifth, we expect significant differences in the irrational belief-distress association depending on whether general versus specific irrational beliefs were measured [21]. Sixth, we expect the association between irrational beliefs and distress would be significantly stronger in studies where irrational beliefs were not only measured occurring naturally but also manipulated experimentally (e.g. instructions to repeat irrational beliefs [22], and randomized clinical trials that included REBT as a treatment group and measured irrational beliefs as a central mechanism of change [23]). Seventh, we expect the irrational belief-distress association will significantly differ depending on the time lag between the measurement of the two constructs [21]. Moreover, the association between irrational beliefs and distress may vary depending on the level of stress potentially being induced by particular events [7]; it is assumed that the level of induced stress may also be maximal when the stress is real and personally relevant [7, 24]. The strength of the irrational belief-distress association may also vary depending on characteristics of specific samples (e.g. participants' age [25]). Researchers' conflicts of interest may systematically impact the (predictive) validity of irrational beliefs, i.e. developers or validators of a particular irrational belief measure may be more motivated to demonstrate a high predictive validity of the measure examined. When (i.e. publication year) and where (i.e. publication country) a re- 
spective study was conducted may also influence the relationship between irrational beliefs and psychological distress [26].

\section{Methods}

\section{Selection of Studies}

Potentially relevant studies were queried in the PsycINFO and Medline databases for all years covered through April 2014. We used the following search terms: 'irrational beliefs', 'distress', 'negative feelings', 'negative emotions', 'anxiety', 'depression', 'anger' and 'guilt'. We also examined the reference sections of all articles included. The search procedure led to the identification of 182 records (online suppl. fig. 1; for all online suppl. material, see www. karger.com/doi/10.1159/000441231). After removing duplicates, the remaining studies were analyzed in detail for relevance based on their abstract. Following the exclusion of irrelevant publications (i.e. the screened abstracts indicated that either irrational beliefs or distress were not measured, irrational beliefs were not measured according to the REBT theory, or the authors did not compute an association between irrational beliefs and distress), a total of 89 potentially relevant articles were inspected for relevance based on their full text. Only studies that fulfilled the following criteria were included: (a) assessed irrational beliefs according to the REBT theory; (b) assessed at least one type of psychological distress (e.g. general distress and anxiety), and (c) reported a numerical relationship between irrational beliefs and distress that was amenable to meta-analytic methods. Eighty-three articles were included for analyses (online suppl. fig. 1).

\section{Coding of Studies}

The authors created a coding system based on the theoretical conceptualization of irrational beliefs and psychological distress (online suppl. table 1). All articles were coded by the first author; in addition, a random sample of 10 studies was coded by a research assistant. The interrater agreement was high $(r \geq 0.95$ for categorical variables and $r \geq 0.99$ for continuous variables). The few cases with diverging assessments were discussed until a consensus was reached.

\section{Meta-Analytic Procedures}

The correlation coefficient, $r$, was the measure of choice to assess the effect size for most analyses. We used only one effect size from each study for each analysis [27], and we randomly selected one within-study level for each categorical moderator to base our analyses on a fully independent sample [28]. All computations were performed on the basis of Fisher's $\mathrm{z}$ transformation of $\mathrm{r}$ before the sample effect sizes were included in our meta-analysis [29]. The weighted mean effect sizes were converted back to $r$ for interpretive purposes. When interpreting the magnitude of $r$, we adopted Cohen's [30] guidelines: $r$ values of $0.10,0.30$ and 0.50 indicate small, medium and large effect sizes, respectively. In the effect size analyses, we used a random-effect model. All analyses were conducted using the $\mathrm{R}$ statistical software package for metaanalysis 'MAc' [29] and 'metafor' [31]. Heterogeneity was assessed using the $\mathrm{Q}$ and $\mathrm{I}^{2}$ statistics [32]. To identify publication bias, asymmetry was tested based on rank correlation [33] and regression tests [34]. Furthermore, a funnel plot was examined using trim and fill procedures [35].

\section{Results}

\section{Descriptive Characteristics}

Participant and Study Characteristics

The total number of participants across all 83 studies comprising 100 different samples was 16,110 . The weighted mean age by sample size was 29.4 years $(S D=10.45$; range $=12.5-72.5$ years). Sixty-six samples were composed of student populations and 34 samples were composed of populations other than high-school or college students. Twenty-two were clinical samples, and 78 were subclinical or nonclinical samples. The included studies were published between 1972 and 2014. They were conducted in 13 different countries; the first 3 places were occupied by the United States, Romania, and the United Kingdom. Irrational beliefs were manipulated before being measured in only 5 samples. In only 17 samples, a stressful event was present.

Irrational Belief and Psychological Distress Measures

The most frequently used irrational belief measures were the IBT [14] $(\mathrm{k}=23)$, SPB [15] $(\mathrm{k}=18)$, IBS [17] $(\mathrm{k}=15)$ and $A B S[16](\mathrm{k}=15)$. The most frequently used measures for psychological distress were the Beck Depression Inventory [36] $(\mathrm{k}=25)$, the State-Trait Anxiety Inventory [37] $(\mathrm{k}=21)$, the Profile of Mood States [38] $(\mathrm{k}=10)$, and a single item rating (i.e. emotional items rated on a Likert scale; e.g. anxiety and depression; $\mathrm{k}=8$ ).

Irrational Belief and Psychological Distress Types

Irrational belief types were measured as follows: demandingness was measured in 40 samples, awfulizing/ catastrophizing in 22 samples, frustration intolerance/ low frustration tolerance in 24 samples and global evaluation in 22 samples. The psychological distress types were measured as follows: general distress was measured in 26 samples, depression in 47 , anxiety in 44 , anger in 17 , and guilt in 6 .

\section{Relationship between Irrational Beliefs and Overall Psychological Distress}

The overall effect of the unconditional model analysis $(\mathrm{k}=100)$ was $\mathrm{r}=0.38(95 \% \mathrm{CI}=0.34-0.42)$. There was significant heterogeneity in the effect sizes $(\mathrm{Q}=904.88$, $\mathrm{p}<0.001 ; \mathrm{I}^{2}=89 \%, 95 \% \mathrm{CI}=0.34-0.42$ ). Significance tests of asymmetry indicated that publication bias was not present in the included studies $(\mathrm{p}>0.22)$. The trim and fill procedure [34] estimated that the number of missing studies needed to attain complete symmetry was one; the imputed study is depicted in the online supplementary 
Table 1. Overall effect sizes for different psychological distress types

\begin{tabular}{lrlllll}
\hline Distress type & $\mathrm{k}$ & $\mathrm{n}$ & $\mathrm{r}$ & $95 \% \mathrm{CI}$ & $\mathrm{Q}$ & $\mathrm{I}^{2}$ \\
\hline General & & & & & & \\
$\quad$ distress & 26 & 4,290 & $0.36^{* *}$ & $0.27,0.44$ & $243.2^{* *}$ & $89 \%$ \\
Depression & 47 & 8,278 & $0.33^{* *}$ & $0.26,0.39$ & $463.06^{* *}$ & $90 \%$ \\
Anxiety & 44 & 5,911 & $0.41^{* *}$ & $0.31,0.5$ & $752.99^{* *}$ & $94 \%$ \\
Anger & 17 & 3,046 & $0.25^{* *}$ & $0.17,0.32$ & $72.86^{* *}$ & $76 \%$ \\
Guilt & 6 & 1,270 & $0.29^{*}$ & $0.02,0.52$ & $122.15^{* *}$ & $95 \%$ \\
\hline
\end{tabular}

$\mathrm{k}$ is the number of effect sizes included in each analysis. ${ }^{*} \mathrm{p}<$ $0.05,{ }^{* *} \mathrm{p}<0.001$.

figure 2. The distress measure was not a significant moderator of the relationship between irrational beliefs and psychological distress on the overall sample ( $p>0.05)$.

\section{Relationship between Irrational Beliefs and}

\section{Psychological Distress Types}

The relationship between irrational beliefs and each distress type reached statistical significance, with anxiety showing the highest association (table 1). Moreover, there was significant heterogeneity for each distress type. Therefore, we tested potential moderators for each distress type.

\section{Moderation Analyses within Distress Types}

Irrational belief measure moderated the association between irrational beliefs and depression as well as the association between irrational beliefs and anxiety ( $\mathrm{p}<$ 0.05), with some measures (e.g. IBT and ABS) showing significantly higher associations than other measures (e.g. SPB; table 2). For an analysis of direct contrast of measures in anxiety and depression, see online supplementary table 2 . The type of irrational beliefs was not a significant moderator of the irrational belief-distress association for depression and anxiety ( $p>0.05)$. However, due to the observed variation among the irrational beliefdistress association for different irrational belief types (with frustration intolerance showing the highest association), we computed an exploratory analysis contrasting frustration intolerance and all other irrational belief types for each psychological distress type. We obtained a significant moderation effect for frustration intolerance versus all other irrational belief types in depression, anxiety, anger, and guilt $(\mathrm{p}<0.05)$, but not in general distress $(p>0.05)$. As shown in table 2, the relationship between frustration intolerance and distress was significantly higher than the relationship between all other irrational belief types combined and distress.

Regarding stressful events, we obtained significant results in the case of depression $(\mathrm{p}<0.05)$; the association between irrational beliefs and depression was higher when a stressful event was present $(\mathrm{r}=0.67, \mathrm{p}<0.001$, $\mathrm{k}=2)$ versus when not $(\mathrm{r}=0.30, \mathrm{p}<0.001, \mathrm{k}=42$; table 2$)$. We also obtained a significant result for general distress $(\mathrm{p}<0.05)$; a higher association between irrational beliefs and general distress was reported when the stressful event was experimentally induced $(\mathrm{r}=0.55, \mathrm{p}<0.001, \mathrm{k}=2)$ versus when the stressful event was real/naturalistic $(\mathrm{r}=$ $0.32, \mathrm{p}<0.001, \mathrm{k}=7$ ). We did not obtain a significant moderation effect for the personal relevance of the stressful event for either anxiety or depression $(p>0.05)$. Age was a significant moderator of the relationship between irrational beliefs and anger; specifically, for every unit increase in age, there was a 0.04 increase in the association between irrational beliefs and anger $(\mathrm{p}<0.05)$. Educational status was also a significant moderator of the association between irrational beliefs and distress in general distress and anger $(p<0.05)$; the association with both general distress and anger was significantly smaller in students $(\mathrm{r}=0.30, \mathrm{p}<0.001, \mathrm{k}=16$ for general distress; $\mathrm{r}=$ $0.19, \mathrm{p}<0.001, \mathrm{k}=14$ for anger) compared with subjects in nonuniversity samples $(\mathrm{r}=0.46, \mathrm{p}<0.001, \mathrm{k}=10$ for general distress; $\mathrm{r}=0.52, \mathrm{p}<0.001, \mathrm{k}=3$ for anger). We did not obtain significant results for depression or anxiety $(p>0.05)$. The clinical status of the participants was a moderator of the association between irrational beliefs and anger $(\mathrm{p}<0.05)$; the association was higher in clinical samples ( $\mathrm{r}=0.54, \mathrm{p}<0.001, \mathrm{k}=3$ ) compared with nonclinical samples $(\mathrm{r}=0.21, \mathrm{p}<0.001, \mathrm{k}=14)$. However, clinical status was not a significant moderator in general distress, depression or anxiety ( $p>0.05)$.

The developer/validator status of the author(s) was a significant moderator in depression and anger $(\mathrm{p}<0.05$; table 2). Notably, the association between irrational beliefs and depression was significantly smaller when any of the authors were a developer/validator $(r=0.22, p<0.001$, $\mathrm{k}=15$ ) versus when none of the authors were a developer/ validator $(\mathrm{r}=0.38, \mathrm{p}<0.001, \mathrm{k}=32)$. Similarly, the association between irrational beliefs and anger was significantly smaller when an author was a developer/validator $(\mathrm{r}=0.15, \mathrm{p}<0.05, \mathrm{k}=7)$ versus when not $(\mathrm{r}=0.33, \mathrm{p}<$ $0.001, \mathrm{k}=10)$. The developer/validator status of the author(s) did not moderate the association between irrational beliefs and psychological distress for either general distress or anxiety $(\mathrm{p}>0.05)$. As can be observed in table 2, we did not obtain significant results for the follow- 
Table 2. Moderation analyses within distress types

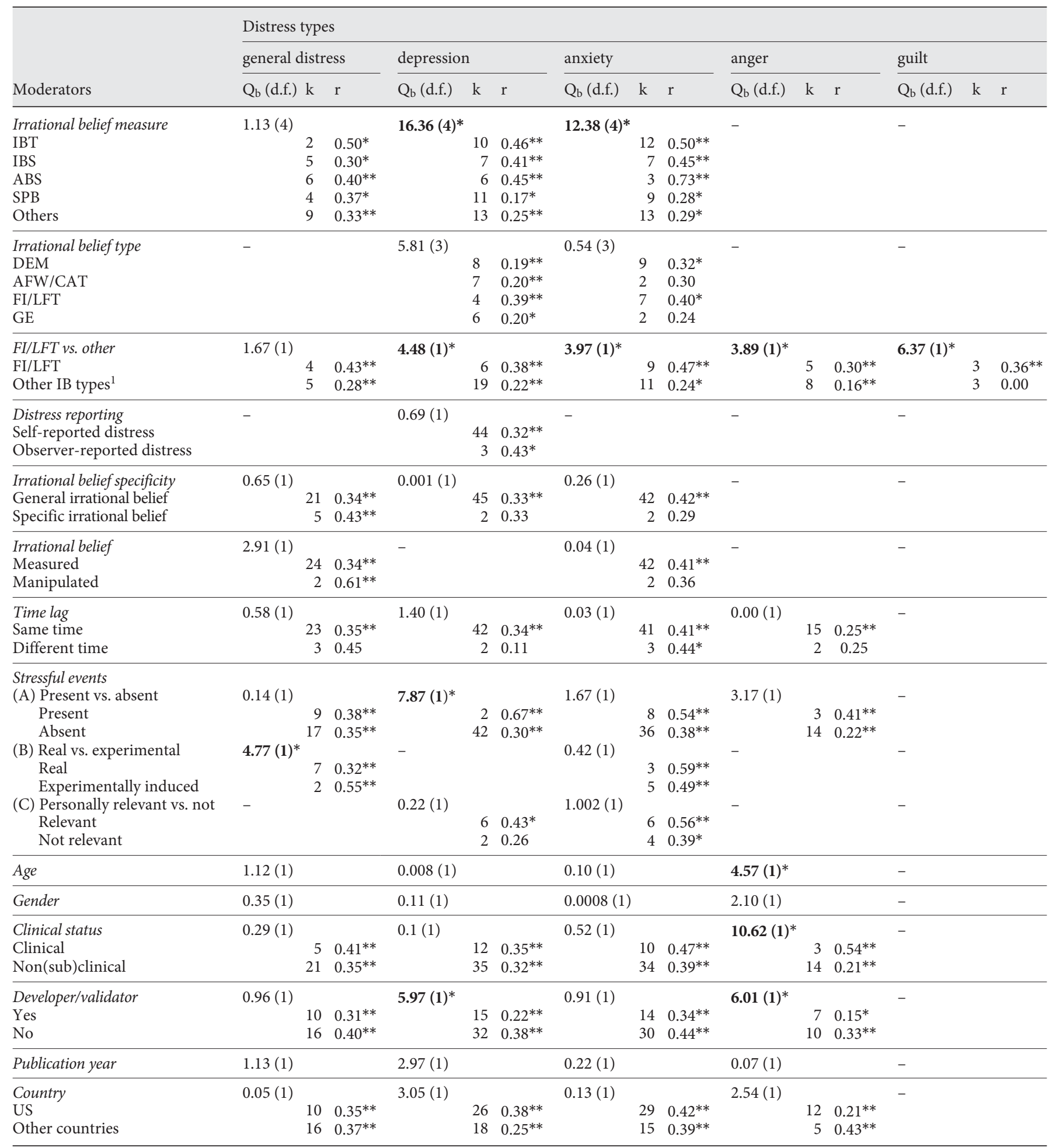

$\mathrm{k}$ is the number of effect sizes included in each analysis. Others = Other instruments used to measure irrational beliefs beside the core ones; DEM = demandingness; $\mathrm{AWF} / \mathrm{CAT}=$ awfulizing/catastrophizing; FI/LFT $=$ frustration intolerance/low frustration tolerance; $\mathrm{GE}=$ global evaluation. ${ }^{1} \mathrm{Demandingness}$, awfulizing/catastrophizing, and global evaluation. ${ }^{*} \mathrm{p}<0.05,{ }^{* *} \mathrm{p}<0.001$. 
ing proposed moderators: self-reported versus observerreported distress, general/core versus specific irrational beliefs, naturally varying versus manipulated irrational beliefs, time lag between irrational beliefs and distress assessment, gender, publication year and publication country $(\mathrm{p}>0.05)$.

\section{Discussion}

The present meta-analysis empirically tested the association between irrational beliefs and psychological distress. The study was based on 100 independent samples of 83 primary studies being conducted in 13 different countries over the last 60 years. Overall, our results corroborate a moderate (overall $\mathrm{r}=0.38$ ) but robust relationship between psychological distress and irrational beliefs. Some authors $[39,40]$ have warned against conducting meta-analyses that include highly heterogeneous populations. Despite including heterogeneous studies reflecting different populations with the aim of avoiding data fragmentation, the present meta-analysis also reported overall effects for the association with different emotional disturbances and conducted moderation analyses within these specific categories. The following variables were significant moderators of the relationship between irrational beliefs and different types of psychological distress: the irrational belief measure chosen in a particular study, irrational belief types (specifically frustration intolerance), stressful events providing the context of assessment, age, educational status, clinical status, and the developer/validator status of the author.

The distress measure did not significantly moderate the association between irrational beliefs and psychological distress. Nonetheless, when split into the various types of distress, the irrational belief measure was a moderator for depression and anxiety. These results may be explained by comparing scales that contain affect-related items (e.g. IBT) with scales that do not (e.g. IBS, ABS and SPB), the items of which may artificially inflate the correlation between irrational beliefs and distress [41]. In the same time, these results could also be attributed to high reliabilities of the used measures [18]. In contrast to the chosen measures, the type of irrational beliefs being assessed did not generally moderate the association between irrational beliefs and psychological distress significantly. These results might suggest that irrational beliefs work as a transdiagnostic vulnerability and/or for some of them the self-report measures might not be sensitive enough (e.g. demandingness [24]). However, frustration intolerance

Irrational Beliefs and Psychological Distress emerged to have a significantly higher correlation with all distress types than all other irrational belief types taken together. Further research is needed to clarify the 'microstructure' of this association, i.e. the associations of specific areas of frustration intolerance with certain distressing problems [42]. Overall, these findings are unexpected, given that demandingness was postulated as the core irrational belief in Ellis' theory [7] that leads to the arousal of the other three (i.e. awfulizing, frustration intolerance, and global evaluation). Therefore, either the centrality of demandingness is retired as a viable hypothesis, or new methods to assess demandingness as part of a tacit/implicit process need to be developed in future research [24].

Regarding stressful events providing the context for assessment, we obtained significant results for depression and general distress. Whereas the association between irrational beliefs and depression was higher when a stressful event was present versus absent, a higher association between irrational beliefs and general distress was reported when the stressful event was experimentally induced versus real/naturalistic. The latter finding was unexpected and may be explained by the novelty of the experimentally induced stressful event, which can potentially increase the reported level of distress. Several sample characteristics emerged as significant moderators of the irrational belief-distress association: the association between irrational beliefs and anger increases with age, it was higher in clinical versus nonclinical samples, and the association with anger and general distress was smaller in students versus nonuniversity samples. Each of these associations needs to be replicated in larger samples before further speculation about potential moderators.

There was no indication of publication bias, neither in the overall sample nor in subsamples. In contrast to our hypothesis, the correlation between irrational beliefs and psychological distress was even smaller in studies conducted by researchers who were developers/validators of an irrational belief scale compared with studies conducted by nondevelopers/nonvalidators. This somewhat counterintuitive result may be explained by developers' stricter adherence to scientific standards. It might be that developers/validators of an irrational belief instrument want to counter suspicions raised by what their developers/validators status might imply. At the same time, a significantly smaller association between irrational beliefs and psychological distress in studies conducted by researchers who were developers/validators of an irrational belief scale compared with studies conducted by nondevelopers/nonvalidators might be an indicator of researchers' increased investment in the development of measures

Psychother Psychosom 2016;85:8-15 
of irrational beliefs that are minimally 'contaminated' by emotional items.

Several limitations emerged in the present meta-analysis. First, despite the sporadic experimental evidence included in this meta-analysis, the presented research does not allow for drawing strong conclusions regarding the causality of the relationship between irrational beliefs and psychological distress. Irrational beliefs were manipulated before being measured in only 5 samples. As a consequence, future research will need to conduct longitudinal analyses which will allow for a better understanding of the potentially etiopathogenetic nature of irrational beliefs [24]. Second, the studies included in the meta-analysis were conducted predominantly in a Western cultural context. Therefore, future studies will need to test whether these results are also applicable to other cultural contexts. Third, nearly all studies included in the meta-analysis employed self-reported measures of the constructs; future research may benefit by including measures based on observer ratings and diagnostic interviews to further control for possible self-report biases. Fourth, most studies were conducted in subclinical or nonclinical samples. Fifth, this meta-analysis was focused on the associations between irrational beliefs and psychological distress (in our sample, we found a $\mathrm{k}$ of 5 studies that also reported an association between rational beliefs and psychological distress) and therefore excluded rational belief scales/ subscales. Rational beliefs were originally conceptualized as low scores on irrational belief measures. Chronologically, scales that measure rational beliefs were developed later; therefore, the available number of studies does not yet allow for meta-analytic analyses.

The results of the present meta-analytic review [ref. 15, $17,19-23,25,26,41$ have been included in the meta-analysis] have implications for future research. Future studies may invest in constructing new methods for the assessment of demandingness as a tacit/implicit process, may investigate how specific components of frustration intolerance relate to certain distressing problems, may conduct more experimental studies that induce a (relevant) stressful event and/or manipulate irrational beliefs, and may conduct longitudinal analyses, which will allow for a better understanding of the potentially etiopathogenetic nature of irrational beliefs. Moreover, future research should be conducted in different cultural contexts on different clinical samples and should measure more specific irrational beliefs (e.g. cancer-related irrational beliefs). Finally, future research will need to systematically integrate other potentially relevant variables that may moderate the relationship between irrational beliefs and psychological distress, such as marital status, occupational status, and income of participants.

To conclude, this comprehensive meta-analysis investigated the robustness of the association between irrational beliefs and various types of psychological distress: a test of one of the major basic associations in modern psychology since the cognitive revolution $[2,3]$. The results show that the overall strength of the relationship between irrational beliefs and psychological distress is modest; however, this relationship holds across different samples, measurements, and study design.

\section{Acknowledgments}

This research was supported by the Scientific Exchange Program Grant SCIEX-NMS-CH 12.319 awarded to Andreea Vîslă, SNSF PZ00P1_136937 to Christoph Flückiger, and SNSF PP00P1-144920/1-2 to Martin grosse Holtforth. The authors would also like to thank Corinne Holzer for her valuable help with the coding process.

\section{References}

1 World Health Organization (WHO): The Global Burden of Disease - 2004 Update. Geneva, WHO, 2008.

2 Cratsley K, Samuels R: Cognitive science and explanations of psychopathology; in Fulford K, Davies M, Gipps R, et al (eds): The Oxford Handbook of Philosophy and Psychiatry, ed 1. New York, Oxford University, 2015, pp 413-433.

3 Chomsky N: Universals of human nature. Psychother Psychosom 2005;74:263-268.

4 Ellis A: New approaches to psychotherapy techniques. J Clin Psychol 1955;11:207-260.
5 Ellis A: Reason and Emotion in Psychotherapy. Oxford, Stuart, 1962.

6 Ellis A, Bernard ME: What is rational-emotive therapy (RET); in Ellis A, Bernard ME (eds): Clinical Applications of Rational-Emotive Therapy. New York, Plenum, 1985, pp 1-30.

7 Ellis A: Reason and Emotion in Psychotherapy, revised. Secaucus, Birch Lane, 1994.

8 Beck AT: Cognitive Therapy for Emotional Disorders. New York, International University, 1976.

9 Lazarus RS: Emotion and Adaptation. New York, Oxford University, 1991.
-10 Vîslă A, grosse Holtforth M, David D: Descriptive/inferential cognitive processes and evaluative cognitive processes: relationships among each other and with emotional distress. J Ration Emot Cogn Behav Ther 2015; 33:148-159.

11 David D, Lynn SJ, Ellis A: Rational and Irrational Beliefs. Research, Theory, and Clinical Practice. New York, Oxford University, 2010.

-12 Haaga D, Davison G: An appraisal of rationalemotive therapy. J Consult Clin Psychol 1993; 61:215-220. 


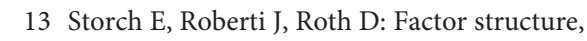
concurrent validity, and internal consistency of the Beck Depression Inventory-Second Edition in a sample of college students. Depress Anxiety 2004;19:187-189.

14 Jones RG: A Factored Measure of Ellis' Irrational Belief Systems with Personality and Maladjustment Correlated. Wichita, Test Systems, 1968.

15 Kassinove H: Self-reported affect and core irrational thinking: a preliminary analysis. J Ration Emot Cogn Behav Ther 1986;4:119-130.

16 DiGiuseppe R, Leaf R, Exner T, Robin MW: The development of a measure of irrational/ rational thinking. The World Congress of Behaviour Therapy, Edinburgh, 1988.

17 Malouff J, Schutte N: Development and validation of a measure of irrational belief. J Consult Clin Psychol 1986;54:860-862.

18 Terjesen M, Salhany J, Sciutto M: A psychometric review of measures of irrational beliefs: implications for psychotherapy. J Ration Emot Cogn Behav Ther 2009;27:83-96.

19 David D, Schnur J, Belloiu A: Another search for the 'hot' cognitions: appraisal, irrational beliefs, attributions, and their relation to emotion. J Ration Emot Cogn Behav Ther 2002;20:93-131.

20 Szentagotai A, Freeman A: An analysis of the relationship between irrational beliefs and automatic thought in predicting distress. J Cogn Behav Psychother 2007;7:1-9.

21 DiLorenzo T, David D, Montgomery GH: The impact of general and specific rational and irrational beliefs on exam distress: a further investigation of the binary model of distress as an emotional regulation model. J Cogn Behav Psychother 2011;11:121-142.
22 Cramer D, Buckland N: Effect of rational and irrational statements and demand characteristics on task anxiety. J Psychol 1995;129:269275.

-23 Szentagotai A, David D, Lupu V, Cosman D: Rational emotive behavior therapy versus cognitive therapy versus pharmacotherapy in the treatment of major depressive disorder: mechanisms of change analysis. Psychotherapy (Chic) 2008;45:523-538.

24 David D, Freeman A, DiGiuseppe R: Rational and irrational beliefs: implications for mechanism of change and practice in psychotherapy; in David D, Lynn SJ, Ellis A (eds): Rational and Irrational Beliefs: Research, Theory, and Clinical Practice. New York, Oxford University, 2010, pp 195-217.

25 Hayslip B Jr, Galt CP, Lopez FG, Nation PC: Irrational beliefs and depressive symptoms among younger and older adults: a cross-sectional comparison. Int J Aging Hum Dev 1994;38:307-326.

26 Kassinove H, Eckhardt CI: Irrational beliefs and self-reported affect in Russia and America. Pers Individ Dif 1994;16:133-142.

27 Cooper H, Hedges LV, Valentine JC: The Handbook of Research Synthesis and MetaAnalysis. New York, Russell Sage Foundation, 2009.

28 Hunter JE, Schmidt FL: Methods of MetaAnalysis: Correcting Error and Bias in Research Findings, ed 2. Thousand Oaks, Sage, 2004.

29 Del Re AC, Hoyt WT: MAc: Meta-Analysis with Correlations 2010. R Package Version 1.0.5. http://CRAN.R-project.org/package! Mac.

30 Cohen J: Statistical Power Analysis for the Social Sciences, ed 2. Hillsdale, Erlbaum, 1988.

-31 Viechtbauer W: Conducting meta-analyses in $\mathrm{R}$ with the metafor package. J Stat Softw 2010; 36:1-48.
2 Higgins J, Thompson S: Quantifying heterogeneity in a meta-analysis. Stat Med 2002;21: 1539-1558.

33 Begg C, Mazumdar M: Operating characteristics of a rank correlation test for publication bias. Biometrics 1994;50:1088-1101.

34 Egger M, Smith G, Schneider M, Minder C: Bias in meta-analysis detected by a simple, graphical test. BMJ 1997;315:629-634.

35 Duval S, Tweedie R: Trim and fill: a simple funnel-plot-based method of testing and adjusting for publication bias in meta-analysis. Biometrics 2000;56:455-463.

36 Beck AT, Steer RA, Brown GK: Beck Depression Inventory. San Antonio, Psychological Corporation, 1996.

37 Spielberger CD, Gorsuch RL, Lushene R, Vagg PR, Jacobs GA: Manual for the StateTrait Anxiety Inventory (Form Y). Palo Alto, Consulting Psychologists, 1983.

38 McNair DM, Lorr M, Droppleman LF: Profile of Mood State. San Diego, Educational and Industrial Testing Service, 1971.

39 Fava GA: Statistical alchemy for drug treatment of generalized anxiety disorder: a commentary on the meta-analysis by Baldwin et al. [BMJ 2011; 342:d1199]. Psychother Psychosom 2011;80:261-263.

40 Fava GA, Guidi J, Rafanelli C, Sonino N: The clinical inadequacy of evidence-based medicine and the need for a conceptual framework based on clinical judgment. Psychother Psychosom 2015;84:1-3.

41 Zurawski R, Smith T: Assessing irrational beliefs and emotional distress: evidence and implications of limited discriminant validity. J Couns Psychol 1987;34:224-227.

42 Harrington N: Frustration intolerance as a multidimensional concept. J Ration Emot Cogn Behav Ther 2007;25:191-211. 